PREPARED FOR THE U.S. DEPARTMENT OF ENERGY, UNDER CONTRACT DE-AC02-76CH03073

PPPL-3550

PPPL-3550

UC-70

Impact of MHD Equilibrium Input Variations on High-beta Stability Boundaries on NSTX

by

F. Paoletti, S.A. Sabbagh, J. Manickam, J. Menard, R.J. Akers, D. Gates, S.M. Kaye, and L. Lao

March 2001

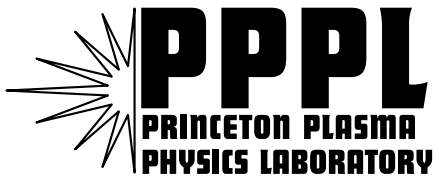

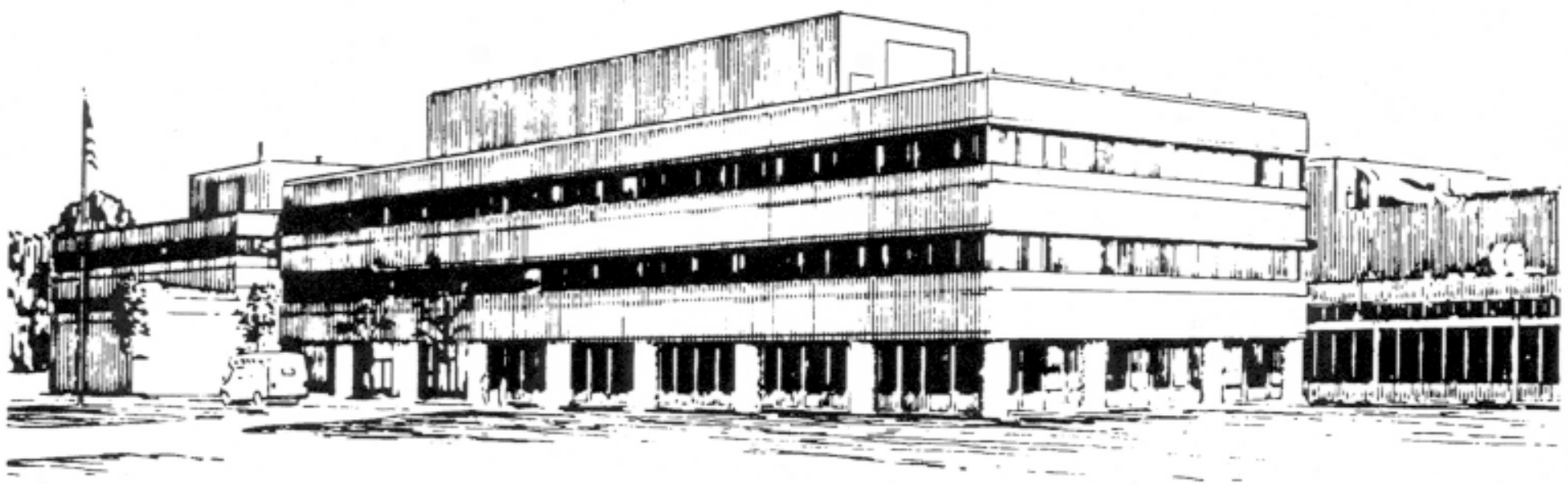

PRINCETON PLASMA PHYSICS LABORATORY PRINCETON UNIVERSITY, PRINCETON, NEW JERSEY 


\section{PPPL Reports Disclaimer}

This report was prepared as an account of work sponsored by an agency of the United States Government. Neither the United States Government nor any agency thereof, nor any of their employees, makes any warranty, express or implied, or assumes any legal liability or responsibility for the accuracy, completeness, or usefulness of any information, apparatus, product, or process disclosed, or represents that its use would not infringe privately owned rights. Reference herein to any specific commercial product, process, or service by trade name, trademark, manufacturer, or otherwise, does not necessarily constitute or imply its endorsement, recommendation, or favoring by the United States Government or any agency thereof. The views and opinions of authors expressed herein do not necessarily state or reflect those of the United States Government or any agency thereof.

\section{Availability}

This report is posted on the U.S. Department of Energy's Princeton Plasma Physics Laboratory Publications and Reports web site in Calendar Year 2001. The home page for PPPL Reports and Publications is: http://www.pppl.gov/pub_report/

DOE and DOE Contractors can obtain copies of this report from:

U.S. Department of Energy

Office of Scientific and Technical Information

DOE Technical Information Services (DTIS)

P.O. Box 62

Oak Ridge, TN 37831

Telephone: (865) 576-8401

Fax: (865) 576-5728

Email: reports@adonis.osti.gov

This report is available to the general public from:

National Technical Information Service

U.S. Department of Commerce

5285 Port Royal Road

Springfield, VA 22161

Telephone: 1-800-553-6847 or

(703) 605-6000

Fax: (703) 321-8547

Internet: http://www.ntis.gov/ordering.htm 


\title{
Impact of MHD Equilibrium Input Variations on the High- Beta Stability Boundaries of NSTX
}

\author{
F. Paoletti ${ }^{1}$, S.A. Sabbagh ${ }^{1}$, J. Manickam², J. Menard², R.J. Akers ${ }^{3}$, D. Gates ${ }^{2}$, \\ S.M. Kaye ${ }^{2}$, L. $\mathrm{Lao}^{4}$. \\ ${ }^{1}$ Columbia University, New York, NY, USA \\ ${ }^{2}$ Princeton Plasma Physics Laboratory, Princeton, NJ, USA \\ ${ }^{3}$ UKAEA Fusion, Culham Science Centre, Oxon, UK \\ ${ }^{4}$ General Atomics, San Diego, CA, USA
}

\begin{abstract}
Ideal MHD stability limits of anticipated plasma configurations for the National Spherical Torus Experiment (NSTX) [Ono, M., et al., Nucl. Fus. 40 (2000) 557] and the dependence on the parameters defining the MHD equilibrium are evaluated. The study provides a quantitative computational evaluation of the stability limit variations induced by changes to the equilibrium of NSTX high- $\beta$ plasmas. The analysis is based on a reference free-boundary equilibrium with $\beta=41.5 \%$, monotonic safety factor $q$ profile $\left(q_{a}=12.1\right.$; $q_{0}=2.8$ ), and broad pressure profile $p$ (peaking factor $\left.F_{p} \equiv p(0) /\langle p\rangle=1.7\right)$. On this reference target local variation of the plasma boundary, safety factor $q$, and pressure $p$ profiles are imposed. Localized inflection of the outboard plasma boundary, produced by near-field effects from ploloidal shaping field coils, weaken the stability due to the destabilization of high- $n$ ballooning modes. Variation of the $q$ profile at different radial location can also degrade stability. Both experimental profiles from existing tokamaks and spherical torus machines and profiles generated from transport modeling of anticipated neutral beam heated plasmas are used. Degraded stability is found at increasing pressure peaking factor due to the destabilization of $n=1$ kink/ballooning modes. Direct access to the second region of stability is found in certain configurations and, for the entire set of variations considered, the lower calculated $\beta$-limits values are still in the range of $20.0 \%$ without considering the stabilizing effect of the passive conducting structures
\end{abstract}

\section{Introduction}

Low aspect ratio fusion concepts are potentially attractive choices for a future fusion energy reactor [1] or a volume neutron source (VNS) [2] [3]. The characteristic difference between a spherical torus (ST) device and a standard tokamak is its small aspect ratio $\left(A=R_{0} / a\right.$ where $R_{0}$ is the plasma major radius; $a$ is its minor radius). A fusion power yield suitable for a reactor is achievable in regimes of high toroidal $\beta$ ( $\beta \equiv 2 \mu_{0} \int p d V / V B_{0}{ }^{2}$ where $B_{0}$ is the vacuum field at the mid-plane half width; $p$ and $V$ are the plasma pressure and volume respectively) combined with high normalized beta $\left(\beta_{N} \equiv \beta\right.$ a $\left.B_{0} / I_{p}\right)$. These two conditions are both requirements since the economic attractiveness of an ST fusion reactor will also require an elevated bootstrap current fraction [4].

The National Spherical Torus Experiment (NSTX) $[5,6]$ has been designed to achieve stable plasma discharges in the mega-ampere current regime at elevated $\beta$ and $\beta_{N}$, exploring regimes of high bootstrap current fraction in the ST geometry.
Previous numerical studies have provided analysis of the ideal MHD stability limits in the low $A$ regimes [7]. In these studies, high- $\beta$ optimized plasma configurations with high bootstrap current fraction were proposed for NSTX and their ideal stability limits were analyzed based on fixed-boundary MHD equilibria. The goal of the present study is to provide a quantitative evaluation of the changes induced on ideal-MHD stability boundaries by variations of the high- $\beta$ equilibria anticipated for NSTX experimental plasmas. In this analysis, a reference equilibrium configuration is defined using the free-boundary EFIT code [8]. Starting from this configuration, the impact of the details of the plasma boundary shape on ideal-MHD stability are studied. In addition, variation of $q$ (safety factor) and $p$ profiles are imposed and their effect on ideal $\beta$-limits is evaluated. Variations on the equilibrium inputs are introduced without imposing the further constraint on the bootstrap current fraction.

Numerical calculations are performed using the high- $n$ ballooning code STBAL [9] and the low$n$ stability code PEST [10]. The fixed-boundary 
EQGAC [11] equilibrium code is also used in conjunction with EFIT to refine the equilibrium solution and the output is used for stability analysis.

The paper is organized as follows. In Section 2., the effect of local indentations in the boundary shape, caused by near-field effects from the poloidal field shaping coil set, on the stability properties of NSTX high- $\beta$ plasmas is shown. Localized boundary indentations produce a weakening of the local magnetic shear in regions of high pressure gradients. These configurations are found to be unstable to incompressible high- $n$ ballooning displacements. A discussion of the role of relevant terms in the ballooning equation is presented.

In Section 3., a numerical evaluation of variation of the ideal $\beta$-limits induced by local changes of $p$ and $q$ profiles is presented. A unique aspect of this study is the use of experimental pressure profiles from other tokamak devices (the spherical torus START [12] and the DIII-D [13] tokamak) as a realistic specification of this free function, and to compare to previous studies which used generic pressure profiles. In addition, pressure profiles obtained using the transport analysis code TRANSP [14] for simulated NSTX neutral-beam heated plasmas with modeled thermal profiles were considered. Variation of the $q$ profile uncovered possible scenarios where direct access to second stability could be achieved. Conclusions are drawn in Section 4.

\section{Impact of Plasma Boundary Defor- mation on Stability Limits in High- $\beta$ Configurations}

The baseline marginally stable reference scenario used in this study is a $\beta=41.5 \%\left(\beta_{N}=8.2\right)$ equilibrium that was constrained to have a high bootstrap current fraction $(\approx 77 \%)$ that was well-aligned with the total current profile [7]. The main plasma parameters of this equilibrium are: $R=0.86 \mathrm{~m} ; a=0.67 \mathrm{~m}$; $k=2.0 ; \delta=0.45 ; I_{p}=1 \mathrm{MA} ; B_{0}=0.3 \mathrm{~T} ; q_{a}=12.1$; $q_{0}=2.8$. This equilibrium uses a set of passive conducting plates for stability. In Figure 1, a plot of the poloidal cross section of the free-boundary plasma as computed using EFIT is shown. The profiles of the current density $J$, the $q$ profile, and the $p$ profile are also shown in Figure 1. The effect of the details of the plasma boundary on the stability property of these kind of configurations was not previously recognized since fixed-boundary equilibria were used to perform stability calculations [7]. In this study, a complement

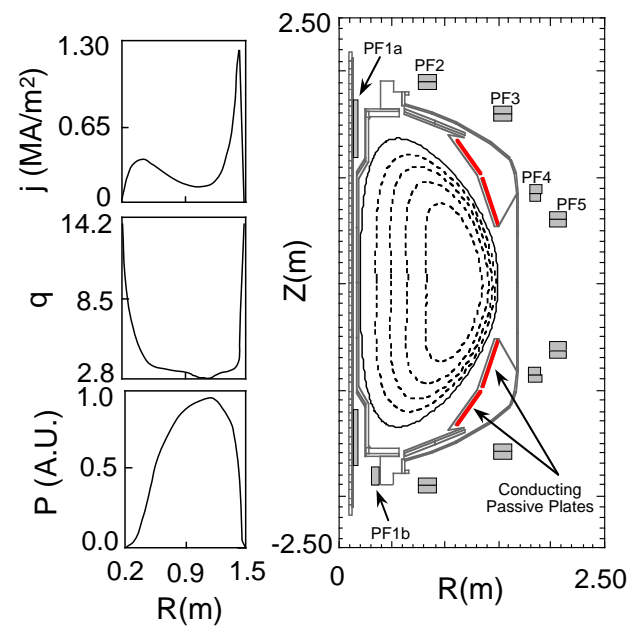

Figure 1. Free boundary poloidal flux contours and $j(R), q(R), P(R)$ profiles for the reference $\beta=41.5 \%$ NSTX case. Also shown are the poloidal field shaping coils and the passive stabilizing structure in the device

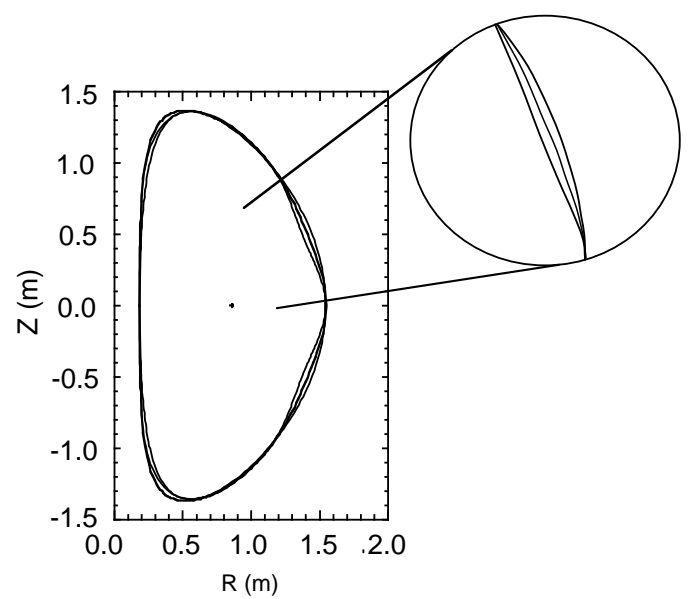

Figure 2. Overlay of the plasma boundaries of two perturbed cases compared with the reference boundary of Figure 1. The local indentations of the perturbed cases are of $5.6 \mathrm{~cm}$ and $2.8 \mathrm{~cm}$ respectively.

to previously obtained results is provided using freeboundary equilibria in a realistic geometry. During the design phase of the NSTX, studies aimed at finalizing the arrangement of the poloidal field coils set (shown in Figure 1) were performed. This was done to find a configuration allowing the sustainment of plasmas with boundary shapes that met the requirements for stability at elevated $\beta$. In one set of these variations, the radius of the $\mathrm{PF} 4$ shaping coils was varied. As the distance between the shaping field coils and the plasma was reduced, changes in the shape of the plasma boundary were observed. Near field effects due to the proximity of the magnetic field 


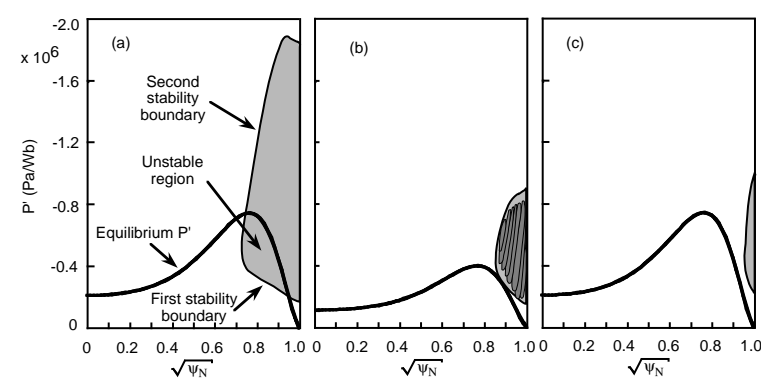

Figure 3. High- $n$ ballooning critical pressure gradient regions for the case with local boundary indentation of $5.6 \mathrm{~cm}$ shown in Figure 2; the unstable regions are shown with a shaded background. In frame (a) the $\beta$ value is the same as the reference case while (b) is the marginally stable case at $\beta=21.2 \%$. The "filament-like" unstable regions (shaded with a darker background) correspond to the off-mid-plane modes contribution. Frame (c) shows the critical pressure gradient plot for the reference case without indentation.

sources led to the generation of localized indentations of the plasma surface. In Figure 2, two of these cases, one more extreme with a local indentation of $5.6 \mathrm{~cm}$ and one intermediate with local indentation of $2.8 \mathrm{~cm}$ are compared. The case with local indentation of $5.6 \mathrm{~cm}$ was obtained using the actual PF4 coil set (as shown in Figure 1) without powering the more external PF5 shaping coils. The boundary of the base configuration is also shown in Figure 2 for reference and it is obtained powering the PF5 coil set while the PF4 pair is not used. From an operational point of view, a combined use of the PF4 and PF5 shaping coil sets widens the accessible operating space of the machine and could be beneficial to the control of ELMs as it was done on the DIII-D tokamak using increased poloidal squareness [15].

Ideal MHD analysis of these cases show that the stability is degraded by the presence of the localized boundary indentations (see Figure 2). Results of the high- $n$ ballooning calculations for the case with larger local indentation are summarized in Figure $3(\mathrm{a}, \mathrm{b}, \mathrm{c})$. The plot shows the critical pressure gradients vs. a minor-radial coordinate $\sqrt{\psi_{N}}$, where the normalized poloidal flux is defined as $\psi_{N} \equiv$ $\left(\psi-\psi_{0}\right) /\left(\psi_{a}-\psi_{0}\right) ;\left(\psi_{0}\right.$ and $\psi_{a}$ are the poloidal flux at the center and at the plasma edge respectively). In the plot is also drawn the profile of the equilibrium $p^{\prime} \equiv \frac{d p(\psi)}{d \psi}$. The shaded region represents the portion of the space $\left(p, \sqrt{\psi_{N}}\right)$ where the eigenvalues $\omega^{2}$ of the high- $n$ ballooning equation are negative, there-

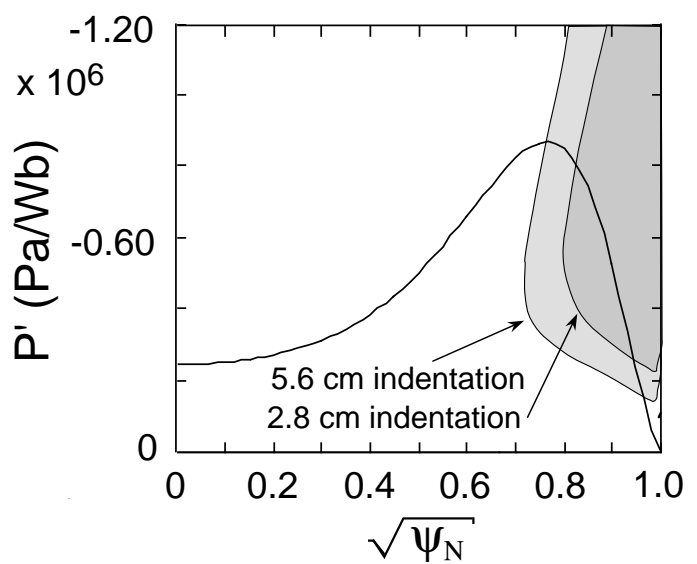

Figure 4. Comparison between the high- $n$ ballooning critical pressure gradients regions for the two cases shown with indented boundaries shown in Figure 2.

fore the plasma is unstable on flux surfaces whose $p$ ' values lie inside the unstable region. In the case of Figure 3(a), the unstable mode is localized in the interval $0.75 \leq \sqrt{\psi_{N}} \leq 0.95$.

By scaling the peak value of $p$ and preserving the shape of the profile, a new equilibrium is generated at lower $\beta(\beta=21.2 \%)$ which is marginally stable to ballooning modes on the first-stability boundary. The $\left(p, \sqrt{\psi_{N}}\right)$ contour plot of critical pressure gradients for this case is shown in Figure 3(b). Since the boundary perturbation is localized off the equatorial plane, the role of ballooning modes maximizing off the mid-plane region, and the impact on the calculated $\beta$-limits was studied. The filament-like formations, highlighted in Figure 3(b) with a darker background, are the unstable regions in the space $\left(p, \sqrt{\psi_{N}}\right)$ for a range of integration origin angle $\vartheta_{0}$ values in the intervals $20.05^{\circ} \leq \vartheta_{0} \leq 40.07^{\circ}$; $-40.07^{\circ} \leq \vartheta_{0} \leq-20.05^{\circ}$ with increments of $\vartheta_{0}=$ $2.86^{\circ}$. For values outside these $\vartheta_{0}$ intervals no further unstable region is found. The marginal $\beta$-limit previously calculated is therefore not changed by the presence of modes with finite radial wavenumbers.

For comparison, Figure 3(c) contains the critical pressure gradient plot for the reference stable case of Figure 1. Progressive reduction of the local boundary deformation reduces the extent of the unstable region as shown in Figure 4 for the two equilibria of Figure 2.

The physics mechanism governing this kind of behavior can be understood considering the role played by the local magnetic shear $S_{\text {local }} \equiv \frac{B \times \nabla \psi}{|\nabla \psi|^{2}} \bullet$ $\nabla \times \frac{B \times \nabla \psi}{|\nabla \psi|^{2}}$ (which should not be confused with the 

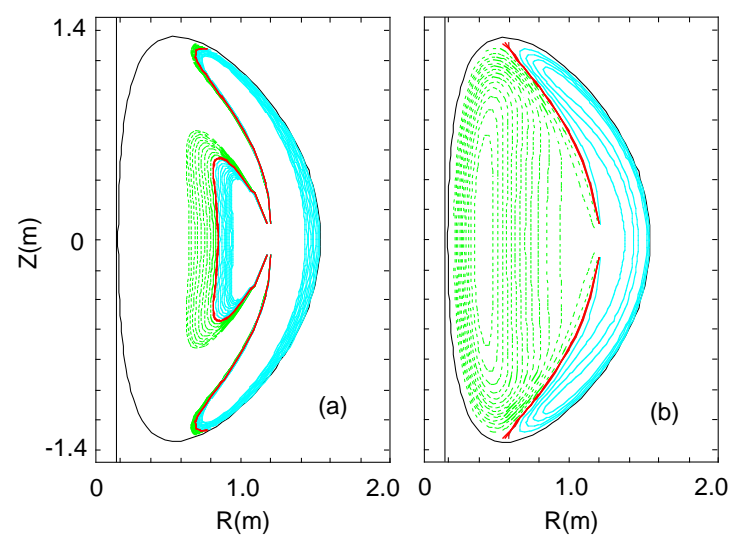

Figure 5. Contour plots of (a) local magnetic shear $S_{l o c a l}$ and (b) $\nabla p \kappa_{n}$ for the reference case of Figure 1. Negative quantities are plotted using a dashed line; positive values are plotted using a solid line; the separation between positive and negative regions is highlighted with a darker solid line.

shear of the $q$ profile i.e., $\left.S_{q} \equiv \frac{d q(\psi)}{d \psi}\right)$ and the normal magnetic field curvature $\kappa_{n}$ in the stability of high$n$ ballooning modes [16]. In general, the sign of $\kappa_{n}$ determines stabilty $\left(\kappa_{n}<0\right.$; good curvature region) or instability $\left(\kappa_{n}>0\right.$; bad curvature region) while large values of $\left|S_{\text {local }}\right|$ in the bad curvature region of the plasma are stabilizing. One of the driving terms in the ballooning equation [17] is the scalar product of the pressure gradient with the normal field curvature $\nabla p \kappa_{n}$. When $\nabla p \kappa_{n}$ is large and $\left|S_{\text {local }}\right|$ is small for $\kappa_{n}>0$ it contributes values towards instability along the ballooning equation integration path. At low aspect ratio, such local conditions can be generated imposing local boundary indentations on the bad curvature region of the plasma.

In the reference equilibrium case we considered, the configuration is stable to high- $n$ ballooning modes. A contour plot of the quantities $\nabla p \kappa_{n}$ and $S_{\text {local }}$ is shown in Figure 5(a) and 5(b) respectively. As local arbitrary deformations are imposed to the plasma boundary in the bad curvature region of the plasma, regions of low $S_{\text {local }}$ are generated on both sides of the deformation. Figure 6(a) shows a contour plot of $S_{\text {local }}$ for this case. A contour plot of $\nabla p \kappa_{n}$ is shown in Figure 6(b) for the same perturbed equilibrium of Figure 6(a). Comparing Figures 6(a) and 6(b) we observe that the regions of $S_{\text {local }} \approx 0$ overlap regions of maximum $\nabla p \kappa_{n}$, which locally destabilizes high- $n$ ballooning modes.
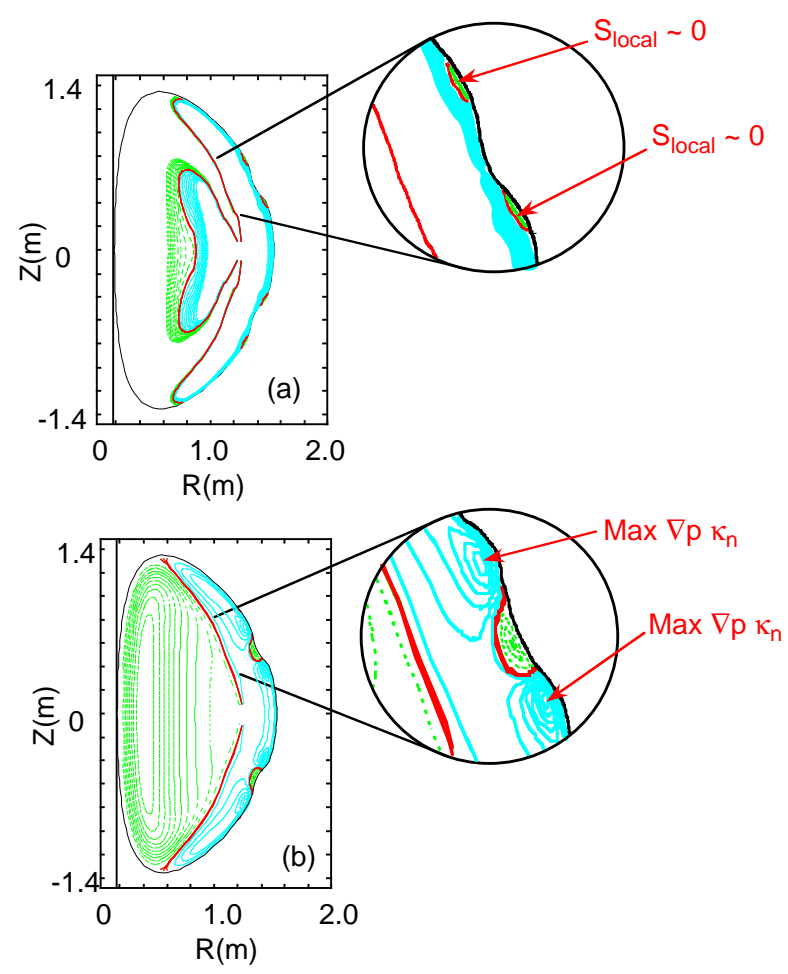

Figure 6. Contour plots of (a) local magnetic shear $S_{\text {local }}$ and (b) $\nabla p \kappa_{n}$ for the case with local boundary indentation of $5.6 \mathrm{~cm}$ shown in Figure 2. The line convention is the same as in Figure 5.

\section{Effect of Localized Profile Varia- tions on Stability of NSTX High- $\beta$ Plasmas}

In this section the effect of profile variations to the stability properties of the high- $\beta$ reference case previously shown is analyzed. The ballooning critical pressure gradients ploted for the reference case are shown in Figure 3(c). This configuration is stable to high- $n$ ballooning modes while the $\beta$-limit is set by an $n=1 \mathrm{kink} /$ ballooning mode which is stabilized by the presence of the passive stabilizing conducting structure. In all low- $n$ kink stability calculations presented in this paper, the NSTX passive conducting structure was modeled as an "effective" conducting wall conformal to the plasma boundary shape placed at $b_{w} \equiv\left(r_{\text {wall }}-a\right) / a=0.25$.

\section{1. $\quad q$ Profile Variations}

Starting from the reference equilibrium, localized variations to the monotonic $q$ profile $\left(q_{0} \simeq 2.8\right)$ were applied. The impact of such variations on the calculated $\beta$-limits were studied keeping the pressure pro- 


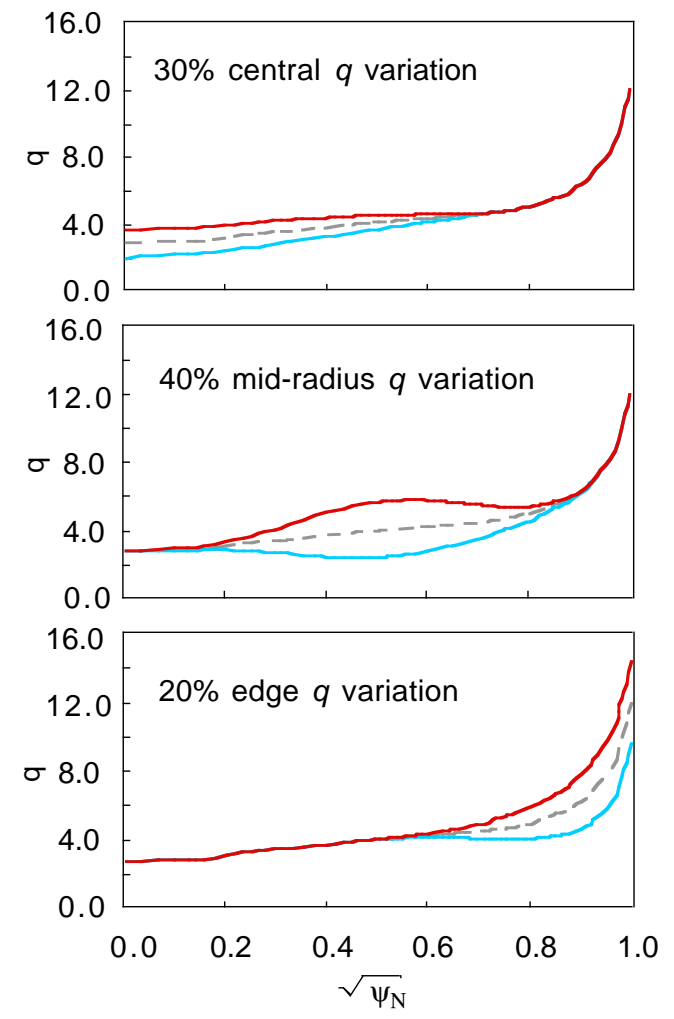

Figure 7. Local $q$ profile variations used in the study (solid lines). The reference $q$ profile is shown with a dashed line.

file shape constant. The $q$ profile was independently varied near the magnetic axis $(+/-30 \%)$, the middle of the minor radius $(+/-40 \%)$, and the plasma edge region $(+/-20 \%)$. The ranges of central and edge variations where chosen to generate profiles with little or no reversed $q$ profile shear $S_{q}$. The effects of strong reversed $S_{q}$ profiles are analyzed in the set of mid-radius variations.

An illustration of the imposed $q$ profile variations is given in Figure 7 . The $\beta$-limits for the most destabilizing set of variations are shown in Table 1 . The $\beta$-limits for all the cases presented in this section are found by scaling down the pressure at fixed profile shape until marginal stability is reached.

Ballooning calculations indicate that the plasma is robustly stable for variations of the central $q$ profile shear $S_{q}$ in the entire range considered. Figure 8(a) and $8(\mathrm{~b})$ show the critical pressure gradient plots for the extremes of the interval at $+30 \%$ and $-30 \% q_{0}$ variation respectively. Low- $n$ kink/ballooning calculations were performed for these cases for modes with $n=1,2,3$ and major departures from the reference case calculated $\beta$-limits are not found in the entire range of $q_{0}$ variations.

\begin{tabular}{|l|l|l|}
\hline $\begin{array}{l}\boldsymbol{q} \text { profile } \\
\text { variation }\end{array}$ & $\begin{array}{l}\beta \text {-limit } \\
\text { no-wall }\end{array}$ & $\begin{array}{l}\beta \text {-limit } \\
\boldsymbol{b}_{w}=\mathbf{0 . 2 5}\end{array}$ \\
\hline $\begin{array}{l}\text { no variation - } \\
\text { reference case }\end{array}$ & $26.5 \%(n=1)$ & $41.5 \%(n=1)$ \\
\hline $\begin{array}{l}(+30 \%) \text { at } \\
\text { center }\end{array}$ & $30.0 \%(n=1)$ & $42.6 \%(n=\infty)$ \\
\hline $\begin{array}{l}(-40 \%) \text { at } \\
\text { mid-radius }\end{array}$ & $17.0 \%(n=1)$ & $36.7 \%(n=\infty)$ \\
\hline $\begin{array}{l}(+20 \%) \text { at } \\
\text { edge }\end{array}$ & $18.5 \%(n=1)$ & $39.2 \%(n=\infty)$ \\
\hline $\begin{array}{l}\text { combination } \\
\text { of last three }\end{array}$ & $16.5 \%(n=1)$ & $32.2 \%(n=\infty)$ \\
\hline
\end{tabular}

Table 1. Calculated $\beta$-limits for the set of $q$ profile variations leading to the largest instability in each radial zone

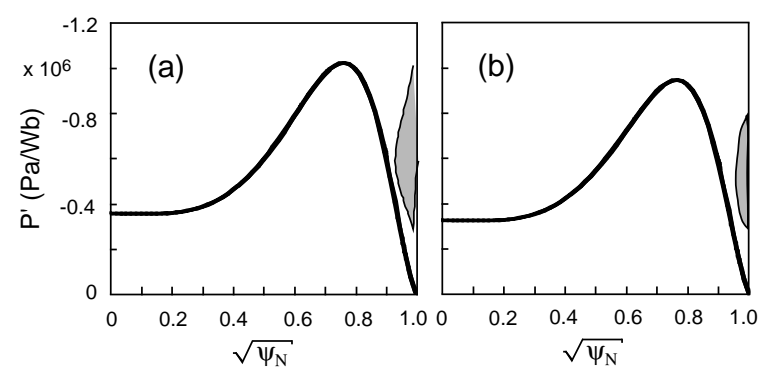

Figure 8. High- $n$ ballooning critical pressure gradient regions for the $+/-30 \%$ central $q$ variation cases (see Figure 7 ) at the same $\beta$ value of the reference case. Frame (a) refers to the positive variation while frame (b) pertains to the negative variation

Increasing $S_{q}$ in the region $0.6 \leq \sqrt{\psi_{N}} \leq 0.9$ (see - $40 \%$ variation at mid-radius) as well as increasing it more near the edge (see $+20 \%$ variation at edge in the interval $0.7 \leq \sqrt{\psi_{N}} \leq 1.0$ ) led to high- $n$ instability in the respective regions. In figures 9 (a) and 9 (b) the high- $n$ ballooning critical pressure gradients plots for the $(+/-40 \%)$ mid-radius $q$ variation are shown respectively. Figures 10(a) and 10(b) pertain to the $(+/-20 \%)$ edge $q$ variation respectively.

The appearance of ballooning unstable regions for increased $S_{q}$ with plasmas operating in the second stability regime can be intuitively understood from the expression that links the local magnetic shear 


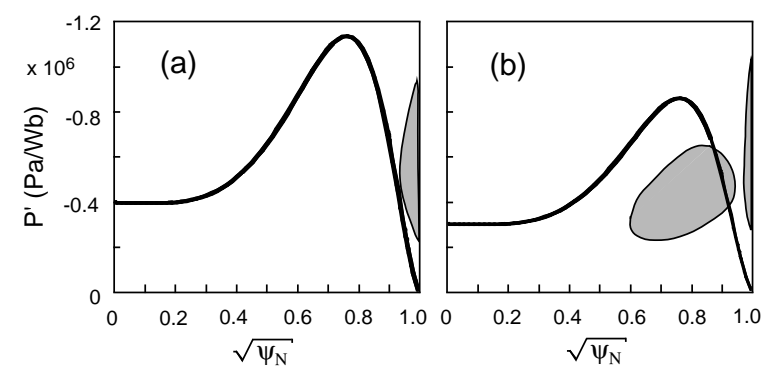

Figure 9. High- $n$ ballooning critical pressure gradient regions for the $+/-40 \%$ mid-radius $q$ variation cases (see Figure 7 ) at the same $\beta$ value of the reference case. Frame (a) refers to the positive variation while frame (b) pertains to the negative variation.

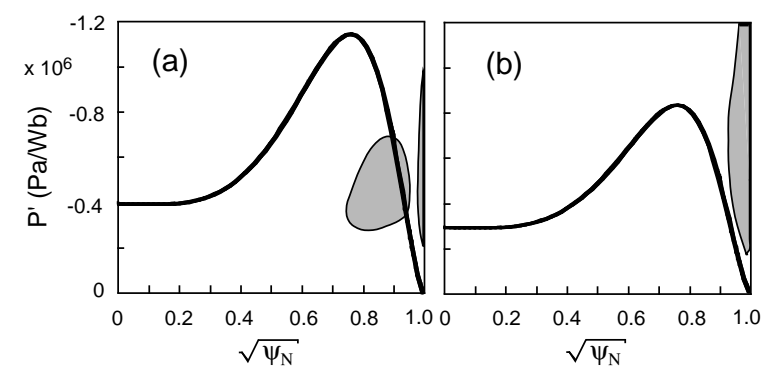

Figure 10. High- $n$ ballooning critical pressure gradient regions for the $+/-20 \% q$ edge variation cases (see Figure 7 ) at the same $\beta$ value of the reference case. Frame (a) refers to the positive variation while frame (b) pertains to the negative variation.

$S_{\text {local }}$ with $S_{q}$ in a standard $(S, \alpha)$ model [17] with no Shafranov shift where, by definition, $S \equiv \frac{r^{2} B_{0}}{q^{2}} S_{q}$ and $\alpha \equiv-\frac{2 \mu_{0} R r q}{B_{0}} \frac{d p(\psi)}{d \psi}$ with $r$ being the minor radial coordinate. In this simple model, the local shear $S_{\text {local }}=S-\alpha \cos \theta$ where $\theta$ is the poloidal angle. In the low- $\beta$ first stability regime $\alpha$ is small and therefore an increased $S$ implies an increased magnitude of $S_{\text {local }}$ which is stabilizing. Conversely, in the high- $\beta$ second stability regime, as in this case, the $\alpha$ term is comparable to $S$ resulting in a small $\left|S_{\text {local }}\right|$ which is destabilizing.

A remarkable feature present in both the $-40 \%$ mid-radius $q$ variation and the $+20 \%$ edge $q$ variation cases is the appearance of a closed unstable region in the $\left(p, \sqrt{\psi_{N}}\right)$ space which intersects the $p$ ' profile. As the pressure is scaled down to reach marginal stability, unlike the normal behavior for a higher aspect ratio tokamak, the unstable region reduces in size until it becomes tangent to the $p$ '

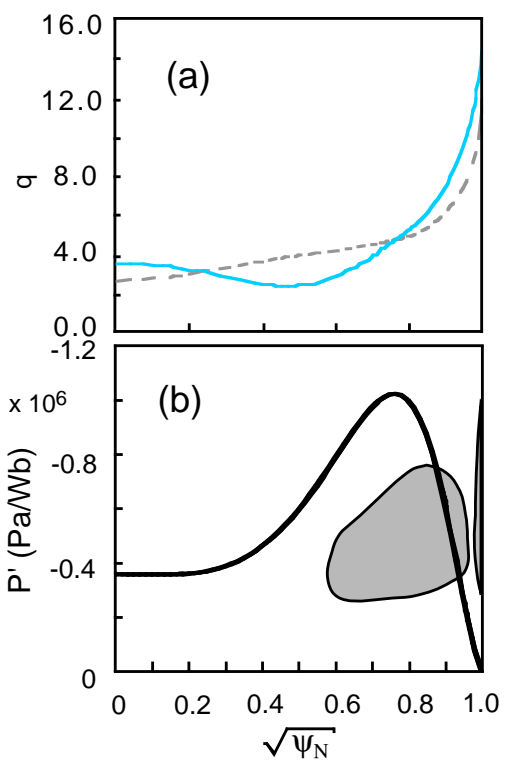

Figure 11. Frame (a) shows a $q$ profile resulting from the combination of the most unstable $q$ variations (solid line). The reference profile is overlaid with a dashed line. Frame (b) shows the high- $n$ ballooning critical pressure gradient regions for the reversed shear $q$ profile case of frame (a) at the same $\beta$ value of the reference case.

curve on the second-stability boundary.

This particular feature could allow, in these configurations, direct access to the second stability region for high- $n$ ballooning modes. In fact, if high- $\beta \mathrm{s}$ are approached at constant pressure profile shape, as $\beta$ is increased the closed unstable region in the space $\left(p, \sqrt{\psi_{N}}\right)$ would appear and grow below the equilibrium $p^{\prime}$ curve until marginal stability is reached. In this case the plasma will always be second stable unless other low- $n$ modes are excited along the way. Low- $n$ kink/ballooning calculations have been performed for the shown scenarios to verify that direct access to the second stable regime is attainable when including the stabilizing conducting wall.

Combining the three $q$ profile variations leading to the largest instability in each radial zone $(+30 \%$ at center, $-40 \%$ at mid-radius, and $+20 \%$ at edge) produces a reversed shear profile, which is marginally stable on the second stability region boundary to high- $n$ ballooning modes at $\beta=32.2 \%$. Figure 11(a) shows the shape of the obtained $q$ profile and compares it to the reference. Figure 11(b) shows the critical pressure gradient plot for this configuration at the same $\beta$ value of the reference case of Figure 1 . At this value of $\beta$, the configuration is unstable. Marginal second-stability is obtained from this case 
Article:

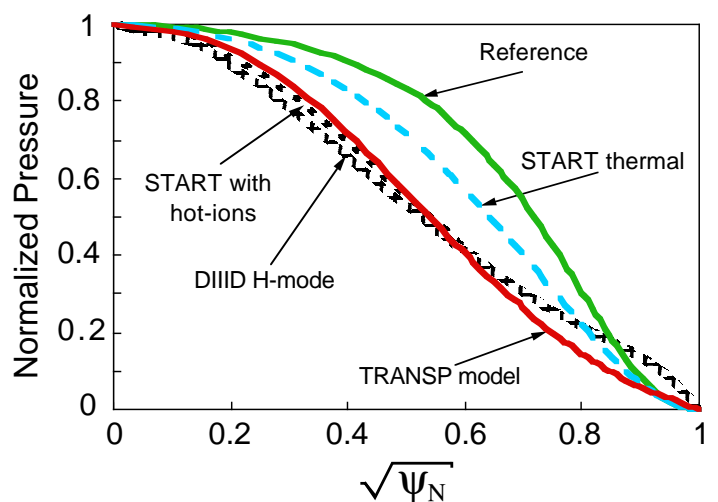

Figure 12. Comparison between the $p$ profiles used in the study. Quantities are normalized to the peak value.

as the pressure is scaled down and the closed unstable region becomes tangent to the equilibrium $p^{\prime}$ curve. Direct access to the second stable regime at constant $p$ profile shape is attainable in this configuration and is not limited by low- $n$ instabilities.

\subsection{Pressure Profile Variations}

In addition to $q$-profile variations, the implementation of different shapes of pressure profiles in the reference plasma configuration shown in Figure 1 was also considered. The pressure profile of the reference case is relatively broad with a pressure peaking factor $F_{p} \equiv P(0) /\langle P\rangle=1.7$. Pressure profile variations were generated by considering both TRANSP modeling performed using the NSTX NBI geometry with modeled kinetic profiles, and experimental profiles from the DIII-D tokamak and the START spherical torus. These variations were considered as they may be more representative of what would be expected in an NBI heated experiment.

In Figure 12, a comparison between the shapes of these profiles is shown. Both calculated and experimental $p$ profiles tend to be more peaked than the reference $p$ profile (START \#35533 thermal [12], $F_{p} \simeq 2.4 ;$ START \#35533 with hot ions [12], $F_{p} \simeq 3.5$; DIII-D \#82205 H-mode [13], $F_{p} \simeq 3.6$; TRANSP-calculated, $F_{p} \simeq 4.2$ ). As shown in the figure, the START thermal component of $p$ is more similar in shape to the reference profile although its peaking factor is higher. As the hot-ion component to the pressure is included the peaking factor of the profile is also increased. Remarkably, a very close similarity between the shapes of the DIII-D H-mode and the START with hot ions contribution pressure profiles was found. This resulted into a nearly iden-

\begin{tabular}{|l|l|l|}
\hline $\begin{array}{l}\boldsymbol{p} \text { profile } \\
\text { variation }\end{array}$ & $\begin{array}{l}\beta \text {-limit } \\
\text { no-wall }\end{array}$ & $\begin{array}{l}\beta \text {-limit } \\
\boldsymbol{b}_{w}=\mathbf{0 . 2 5}\end{array}$ \\
\hline $\begin{array}{l}\text { no variation - } \\
\text { reference case }\end{array}$ & $26.5 \%(n=1)$ & $41.5 \%(n=1)$ \\
\hline START thermal & $26.1 \%(n=1)$ & $40.3 \%(n=1)$ \\
\hline $\begin{array}{l}\text { DIII-D H-Mode } \\
\text { START with hot ions }\end{array}$ & $22.3 \%(n=1)$ & $38.1 \%(n=1)$ \\
\hline TRANSP & $25.0 \%(n=1)$ & $35.9 \%(n=1)$ \\
\hline
\end{tabular}

Table 2. Calculated $\beta$-limits for the set of imposed $p$ profile variations

tical effect on the stability of the studied configurations whether the former or the latter profile shape was used. The calculated (TRANSP) NSTX pressure profile has the highest pressure peaking factor among the set of profiles shown in Figure 12. In the TRANSP calculation, the electron density profile is assumed to be flat $n_{e}(0) /\left\langle n_{e}\right\rangle=1.1$, similar to that expected for H-mode operation. The temperature profiles are calculated assuming this density profile and their peak values are normalized to give $\beta \simeq 40 \%$, after taking the beam contribution to $\beta$ into account.

Previous studies show that the stability of typical low aspect ratio target configurations degrades as $F_{p}$ increases [7]. The NSTX no-wall $\beta$-limits computed for the $p$ profiles with increasing pressure peaking factor in the present study are reduced but remain in the $20 \%$ range regardless of the $q$ profile variation considered. In the following, the results of this analysis are presented.

First, the cases where the different $p$ profile shapes are combined with the reference $q$ profile are examined.

The calculated $\beta$-limits for these cases are summarized in Table 2. Using the START thermal $p$ profile in the NSTX reference case geometry, a slight reduction of the $\beta$-limit $(\approx 40.3 \%)$ is observed in the presence of a conformal conducting wall at $b_{w}=0.25$, as compared to the reference case. The corresponding no-wall limit is at $\beta=26.1 \%$. The behavior in this case is similar to the reference equilibrium and the limiting factor is always due to an $n=1$ kink/ballooning instability.

Of particular interest are cases where the 


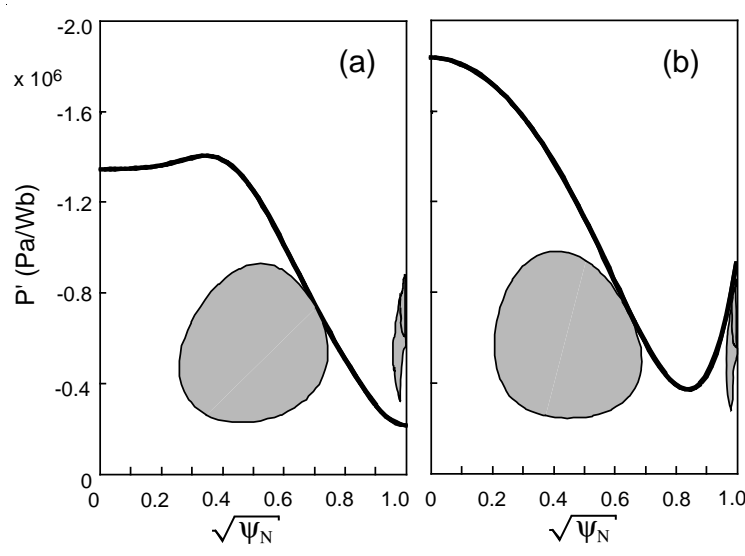

Figure 13. High- $n$ ballooning marginally stable critical pressure gradient regions for the $p$ profile variation cases. Frame (a) refers to the TRANSP $p$ profile case (at $\beta=40.2 \%$ ) and frame (b) to the DIII-D H-mode $p$ profile case (at $\beta=43.3 \%$ ).
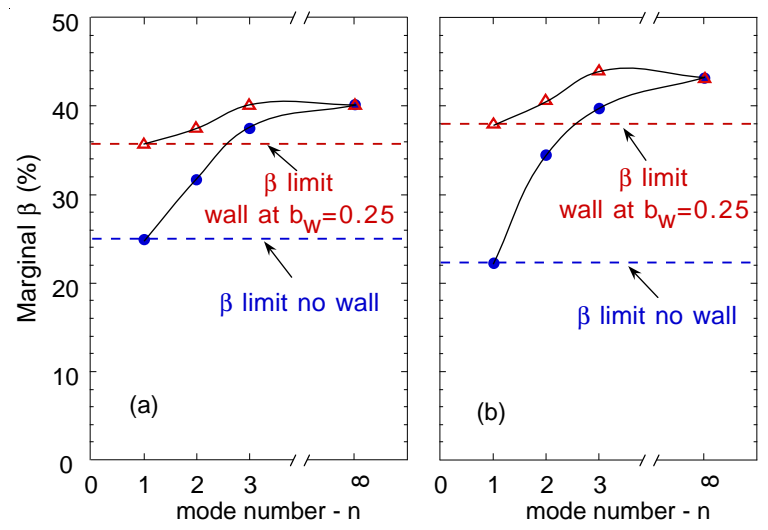

Figure 14. Low- $n$ kink/ballooning computed marginal $\beta$ values vs. toroidal mode number $n$ for the two cases of Figure 13(a), 13(b).

TRANSP $p$ and the DIII-D H-mode $p$ were used in lieu of the reference profile. In Figures 13(a) and $13(\mathrm{~b})$ the high- $n$ critical pressure gradient plots for these two equilibria are compared. Both cases are marginally stable on the second stability boundary but at different values of $\beta(\beta=40.2 \%$ for the TRANSP $p$ and $\beta=43.3 \%$ for the DIII-D Hmode $p$ ). In both cases, though, high- $n$ ballooning instabilities are not the limiting factors, low- $n$ kink/ballooning calculations for $n=1,2,3$ were performed and the results for each case are summarized in Figures 14(a) and 14(b) respectively. In the DIII$\mathrm{D}$ H-mode case the no-wall maximum $\beta$ is limited by an $n=1 \mathrm{kink} /$ ballooning instability to a value of $22.3 \%$. The TRANSP pressure profile has greater total peaking factor, but does not have the relatively large edge pressure gradient of the DIII-D H-mode profile responsible for the increased drive of an external kink/ballooning mode. For the former case, the maximum achievable $\beta$ is also limited by an $n=1$ mode but with a larger $\beta=25.0 \%$ as compared to the latter case.

In both cases, the stabilizing effect due to the presence of the passive plate structure is substantial. For the DIII-D H-mode $p$ profile, the stability limit for the $n=1 \mathrm{kink} /$ ballooning mode increases to $\beta=38.1 \%$. The equilibrium using the TRANSP $p$ profile in the presence the conducting wall yields an $n=1 \beta$-limit of $35.9 \%$. The large difference between the wall and no-wall $\beta$-limits will allow NSTX experiments to identify and study the resistive wall mode (RWM) [18] while operating significantly above the no-wall limit.

In order to understand the effect of the passively conducting system on the internal structure of the modes, the poloidal mode number $(m)$ structure of the destabilized $n=1 \mathrm{kink} /$ ballooning mode have been studied using the PEST code. The results obtained for the DIII-D H-mode $p$ profile case at $\beta=43.3 \%$ are shown in Figures $15(\mathrm{a}, \mathrm{b}, \mathrm{c})$. Figures $15(\mathrm{a})$ and $15(\mathrm{~b})$ pertain to the case without a stabilizing conducting structure. In this configuration, two modes are present, one more characterized by dominant external-kink components (see Figure 15(a)) and another with a larger internalkink structure. The effect of the passive conducting shell on the mode structure can be seen in Figure 15(c) Here, although the external-kink components were stabilized, the internal-kink portion of the mode structure is still present.

The characteristic differences in the mode structure associated with a variation of the $p$ profile shape can be appreciated by comparing the TRANSP and the DIII-D H-mode $p$ profiles cases. At $\beta=26.3 \%$ and without a stabilizing wall, both cases are unstable to $n=1 \mathrm{kink} /$ ballooning modes while only one mode is present. The structure of the mode, though, is different in the two cases. The TRANSP $p$ profile case (more peaked) shows a global mode (see Figure 16(a)), while the DIII-D $p$ profile case (less peaked and with an edge pedestal) has a more predominant external kink structure (see Figure 16(b)). For this reason, the latter class of $p$ profiles is among those that will more likely show a resistive wall mode kind of instability on NSTX therefore providing a suitable target for passive wall stabilization studies [19].

When the variations in the $q$ profile leading to instability are combined with the DIII-D H-mode 

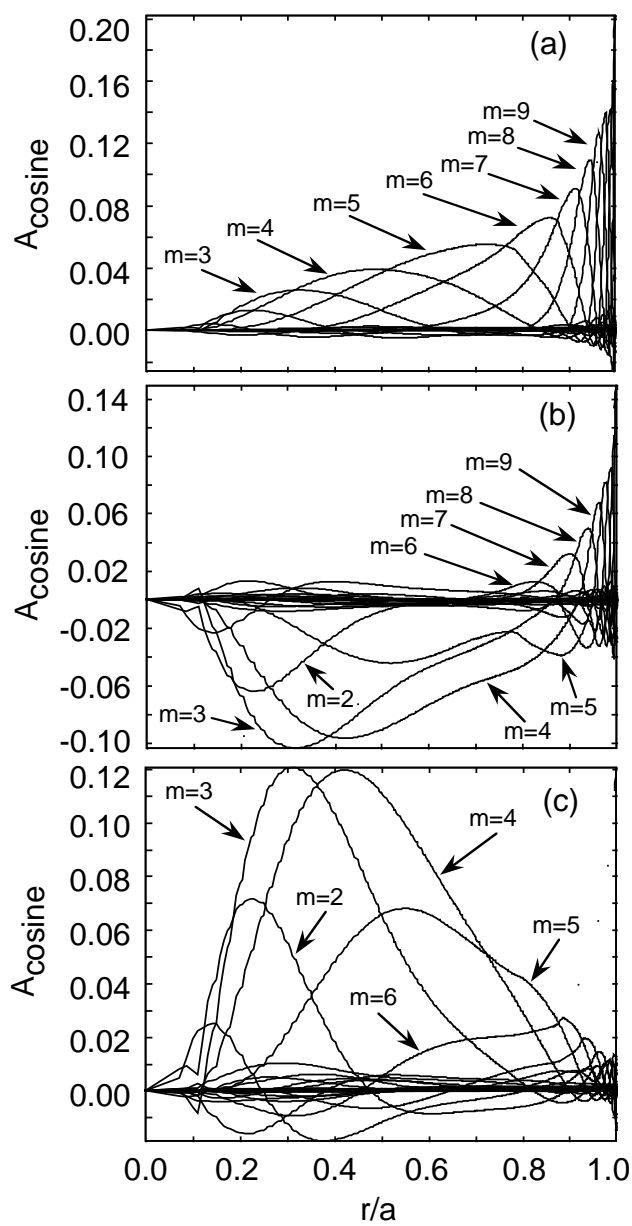

Figure 15. $n=1 \mathrm{kink} /$ ballooning $m$ mode structure (amplitude of the cosine component of the Fourier expansion) for the DIII-D H-mode $p$ profile case of Figure 13. Frames (a), (b) show the structure of the two unstable modes at the no-wall limit. Frame (c) refers to the mode structure of the unstable mode in the presence of a conformal conducting wall $\left(b_{W}=0.25\right)$.

and TRANSP pressure profiles, the $\beta$-limits decrease to values of the order of $20 \%$. The limiting instability in these cases is the high- $n$ ballooning mode, which is unaffected by the presence of a nearby conducting structure. These plasmas are marginally stable on the first stability region boundary.

The "least stable" case was obtained using a certain $q$ profile shape (not included in the previous $q$ profile variation) combined with the TRANSP pressure profile (highest peaking factor). This $q$ profile was obtained from the reference $q$ broadening the profile in the region $0.5 \leq \sqrt{\psi} \leq 0.8$ as shown in Figure 17. This profile choice moves the closed unstable region in the critical pressure gradient space to center on the equilibrium $p^{\prime}$. Figures 18(a) and 18(b)
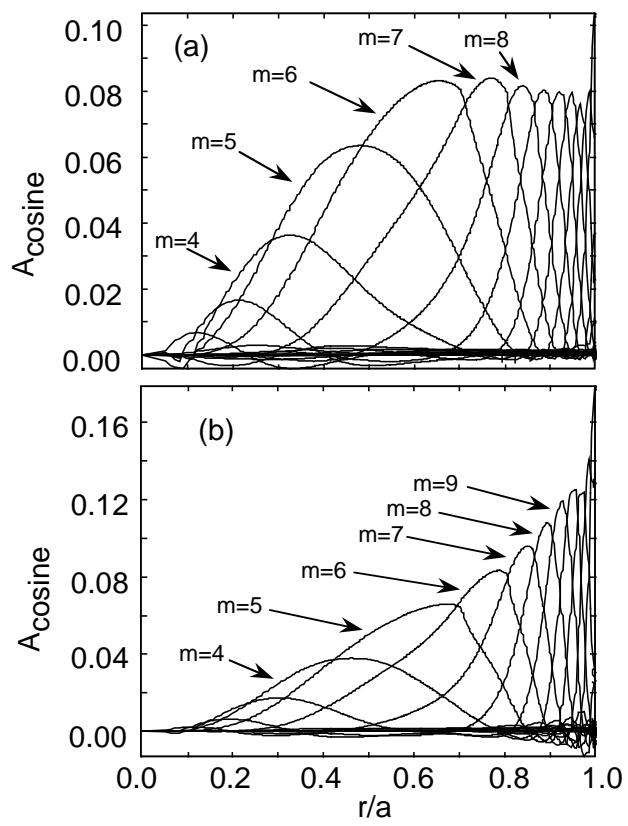

Figure 16. Comparison between the $n=1$ kink/ballooning $m$ mode structure of the TRANSP $p$ profile case (a) and the DIII-D H-mode $p$ profile case (b). Both cases are calculated without a stabilizing conducting wall at $\beta=26.3 \%$.

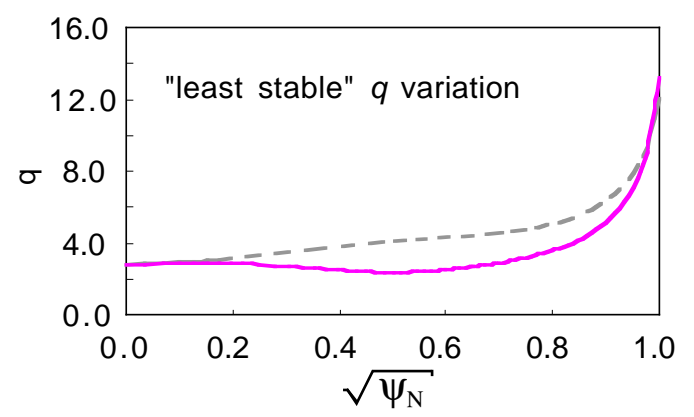

Figure 17. "Least stable" $q$ profile shape obtained from the reference broadening the profile in the region $0.5 \leq$ $\sqrt{\psi} \leq 0.8$ (solid line). The reference case is shown with a dashed line.

show the high- $n$ critical pressure gradient plot for the DIII-D H-mode and TRANSP pressure profiles cases at the same $\beta$ value of the reference configuration. As the pressure is scaled down in both cases, the plasma is still unstable until the closed unstable region completely disappears bringing the configuration to be marginally stable on the first stability boundary. Marginal stability is reached at $\beta$ values of $22.5 \%$ and $19.4 \%$ respectively. 

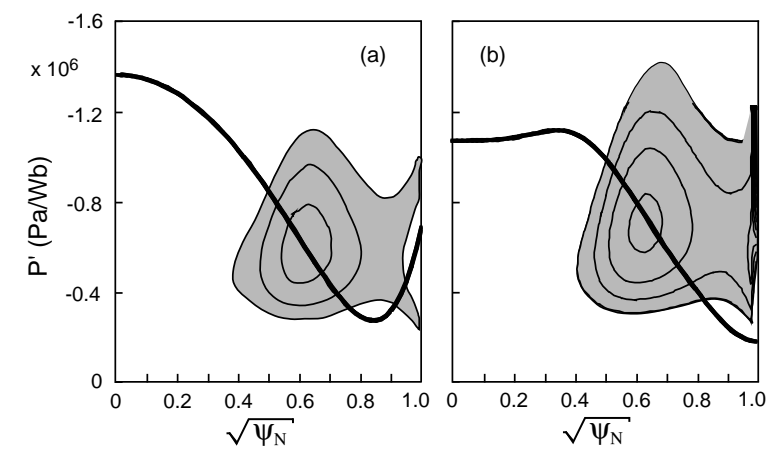

Figure 18. High- $n$ ballooning critical pressure gradient regions for the cases where the $q$ profile of Figure 17 is combined with (a) the DIII-D H-mode $p$ profile and (b) the TRANSP $p$ profile. The value of $\beta$ is the same as in the reference case.

\section{Conclusions}

The behavior of the computed ideal MHD stability boundaries of NSTX as a function of the equilibrium parameters in the high- $\beta$ and high- $\beta_{N}$ regime was studied. Variations of the equilibrium inputs were imposed on a $\beta=41.5 \%\left(\beta_{N}=8.2\right)$ reference case with a monotonic $q$-profile $\left(q_{0} \simeq 2.8\right)$ and a broad pressure profile $\left(F_{p} \equiv p(0) /\langle p\rangle=1.7\right)$. The equilibrium parameters varied included the plasma boundary, $q$, and $p$ profiles.

The impact on stability of local plasma boundary indentations in the outboard portion of the poloidal cross section was analyzed. The boundary deformations weakened the stability of the reference configuration due to the excitation of high- $n$ ballooning modes. Marginal stability for a case with local boundary indentations of $5.6 \mathrm{~cm}$ was found at $\beta=21.2 \%$. The physics responsible for the reduction of high- $n$ mode stability and thereby the reduction of the $\beta$ limit is the weakening of the local magnetic field shear in the proximity of the boundary deformation on the bad curvature side of the poloidal cross section coexisting with an increased product $\nabla p \kappa_{n}$ in the same region. On NSTX, such local indentations of the plasma boundary could be intentionally produced with the use of the existing set of shaping field coil set.

Local perturbations of $q$ from the reference case indicate that the plasma is robustly stable for variations of $q_{0}$ up to $30 \%$. A magnetic $q$ profile shear reduction at mid-radius as well as an increase at the edge degrades stability due to the destabilization of high- $n$ ballooning modes. Direct paths to the second region of stability have also been identified.
Perturbation to $p$ profiles are realized incorporating experimental profiles from the START spherical torus, the DIII-D tokamak, and also using the results of transport analysis code modeling. The implementation of experimental profiles led to reduced $\beta$-limit due to the increased pressure peaking factor with respect to the optimized reference case. Nontheless, this reduction is not as drastic as previous theoretical studies, where simple profile's parametrizations where used, would have predicted.

In conclusion, regardless of the $q$ or $p$ profile variation applied, the lowest calculated no-wall $\beta$-limits remain in the range of $20 \%$. Operation of NSTX significantly above $\beta \approx 20 \%$ should require favorable $q$ profile shapes to avoid the high- $n$ first stability boundary, and conducting wall stabilization for $n=1$ kink/ballooning stability.

\section{Acknowledgements}

We acknowledge J. Bialek, J. Ferron, R. Kaita, H. Kugel, E. Lazarus, M. Ono, M. Peng, N. Pomphrey, and A. Sykes for the useful discussions on these topics and L.M. Carlucci for her technical/editorial comments. Work supported by USDOE Contracts: DE-FG02-99ER54524 (CU), DE-AC02-76CH03073 (PPPL), and DE-AC05-00R22725 (ORNL).

\section{References}

[1] PENG, Y.-K., Phys. Plasmas 7 (2000) 1681.

[2] STAMBAUGH, R. D., CHAN, V. S., MILLER, R. L., and SCHAFFER, M. J., Fus. Technol. 33 (1998) 1.

[3] PENG, Y.-K. M., GALAMBOS, J. D., and SHIPE, P. C., Fus. Technol. 21 (1992) 1729.

[4] Devoto, R. S., FEnSTERMACHER, M. E., and MIRIN, A. A., Nucl. Fus. 29 (1989) 1769.

[5] ONO, M., KAYE, S., PENG, Y.-K., BARNES, G., BLANCHARD, W., et al., Nucl. Fus. 40 (2000) 557.

[6] KAYE, S., ONO, M., PENG, Y.-K., BATCHELOR, D., CARTER, M., et al., Fus. Technol. 36 (1999) 16.

[7] MENARD, J. E., JARDIN, S. C., KAYE, S. M., KESSEL, C. E., and MANICKAM, J., Nucl. Fus. 37 (1997) 595.

[8] LAO, L. L., St. John, H., STAMBAUGH, R. D., KELLMAN, A. G., and PFEIFFER, W., Nucl. Fus. 25 (1985) 1611.

[9] PHILliPS, M. W., TODD, A. M. M., HUGHES, M. H., MANICKAM, J., JOHNSON, J. L., et al., Nucl. Fus. 28 (1988) 1499. 
[10] GRIMM, R., DEWAR, R. L., and MANICKAM, J., J. Comput. Phys. 49 (1983) 94.

[11] DELUCIA, J., JARDIN, S. C., and TODD, A. M. M., J. Comput. Phys. 37 (1980) 183.

[12] AKERS, R. J., APPEL, L. C., CAROLAN, P. G., CONWAY, N. J., COUNSELL, G. F., et al., Ion physics in the "start" spherical tokamak, in 25th European Conference on Controlled Fusion and Plasma Physics, p. 2014, Praha, Chec. Republic, 1998, European Physical Society.

[13] STRAIT, E. J., CASPER, T. A., CHU, M. S., FERRON, J. R., GAROFAlO, A., et al., Phys. Plasmas 4 (1997) 1783.

[14] GOLDSTON, R. J., MCCUNE, D. C., TOWNER, H. H., DAVIS, S. L., HAWRYLUK, R. J., et al., J. Comput. Phys. 43 (1981) 61.

[15] FERRON, J. R., CHU, M. S., JACKSON, G. L., LAO, L. L., MILLER, R. L., et al., Phys. Plasmas 7 (2000) 1976.

[16] GREENE, J. M. and CHANCE, M. S., Nucl. Fus. 21 (1981) 453.

[17] CONNOR, J. W., HASTIE, R. J., and TAYLOR, J. B., Phys. Rev. Lett. 40 (1978) 396.

[18] GArofalo, A. M., TURnBUlL, A. D., AUSTIN, M. E., BIALEK, J., CHU, M. S., et al., Phys. Rev. Lett. 82 (1999) 3811.

[19] GAROFALO, A. M., TURNBULL, A. D., STRAIT, E. J., AUSTIN, M. E., BIALEK, J., et al., Phys. Plasmas 6 (1999) 1893. 


\section{External Distribution}

Plasma Research Laboratory, Australian National University, Australia

Professor I.R. J ones, Flinders University, Australia

Professor J oão Canalle, Instituto de Fisica DEQ/IF - UERJ , Brazil

Mr. Gerson O. Ludwig, Instituto Nacional de Pesquisas, Brazil

Dr. P.H. Sakanaka, Instituto Fisica, Brazil

The Librarian, Culham Laboratory, England

Library, R61, Rutherford Appleton Laboratory, England

Mrs. S.A. Hutchinson, JET Library, England

Professor M.N. Bussac, Ecole Polytechnique, France

Librarian, Max-Planck-Institut für Plasmaphysik, Germany

J olan Moldvai, Reports Library, MTA KFKI-ATKI, Hungary

Dr. P. Kaw, Institute for Plasma Research, India

Ms. P.J . Pathak, Librarian, Insitute for Plasma Research, India

Ms. Clelia De Palo, Associazione EURATOM-ENEA, I taly

Dr. G. Grosso, Instituto di Fisica del Plasma, Italy

Librarian, Naka Fusion Research Establishment, J AERI, J apan

Library, Plasma Physics Laboratory, Kyoto University, J apan

Research Information Center, National Institute for Fusion Science, J apan

Dr. O. Mitarai, Kyushu Tokai University, J apan

Library, Academia Sinica, Institute of Plasma Physics, People's Republic of China

Shih-Tung Tsai, Institute of Physics, Chinese Academy of Sciences, People's Republic of China

Dr. S. Mirnov, Triniti, Troitsk, Russian Federation, Russia

Dr. V.S. Strelkov, Kurchatov Institute, Russian Federation, Russia

Professor Peter Lukac, Katedra Fyziky Plazmy MFF UK, Mlynska dolina F-2, Komenskeho Univerzita, SK-842 15 Bratislava, Slovakia

Dr. G.S. Lee, Korea Basic Science Institute, South Korea

Mr. Dennis Bruggink, Fusion Library, University of Wisconsin, USA

Institute for Plasma Research, University of Maryland, USA

Librarian, Fusion Energy Division, Oak Ridge National Laboratory, USA

Librarian, Institute of Fusion Studies, University of Texas, USA

Librarian, Magnetic Fusion Program, Lawrence Livermore National Laboratory, USA

Library, General Atomics, USA

Plasma Physics Group, Fusion Energy Research Program, University of California at San Diego, USA

Plasma Physics Library, Columbia University, USA

Alkesh Punjabi, Center for Fusion Research and Training, Hampton University, USA

Dr. W.M. Stacey, Fusion Research Center, Georgia Institute of Technology, USA

Dr. J ohn Willis, U.S. Department of Energy, Office of Fusion Energy Sciences, USA

Mr. Paul H. Wright, Indianapolis, Indiana, USA 
The Princeton Plasma Physics Laboratory is operated by Princeton University under contract with the U.S. Department of Energy.

\author{
Information Services \\ Princeton Plasma Physics Laboratory \\ P.O. Box 451 \\ Princeton, NJ 08543
}

Phone: 609-243-2750

Fax: 609-243-2751

e-mail: pppl_info@pppl.gov

Internet Address: http://www.pppl.gov 